ORIENTAL JOURNAL OF CHEMISTRY

An International Open Free Access, Peer Reviewed Research Journal

www.orientjchem.org
ISSN: 0970-020 X

CODEN: OJCHEG

2014, Vol. 30, No. (2):

Pg. 745-753

\title{
Quantum Mechanical Investigation of Bond Gaps of $\Pi$-acceptor Ligand Alone and Affected Nanoring Field: EPR and NMR study
}

\author{
MEHRNOOSH KHALEGHIAN ${ }^{1 *}$, MOHAMMAD YARI ${ }^{1}$, \\ FATEMEH AZARAKHSHI ${ }^{2}$ and GHOLAMREZA GHASHAMI ${ }^{3}$
}

1Department of Chemistry, Islamshahr Branch, Islamic Azad University, Tehran, Iran.

${ }^{2}$ Department of Chemistry, Varamin-Pishva Branch, Islamic Azad University, Varamin, Iran.

${ }^{3}$ Department of Engineering, Islamshahr Branch, Islamic Azad University, Tehran, Iran.

*Corresponding author E-mail: Mehr_khaleghian@iiau.ac.ir, Mehr_khaleghian@yahoo.com

http://dx.doi.org/10.13005/ojc/300247

(Received: March 03, 2014; Accepted: April 04, 2014)

\begin{abstract}
We studied non-bonded interaction of the $\left[\mathrm{Co}(\mathrm{CN})_{6}\right]^{3-}$ complex Situated $\mathrm{B}_{24} \mathrm{~N}_{24}$ nanoring. Early, the geometry of $\left[\mathrm{Co}(\mathrm{CN})_{6}\right]^{3-}$ and $\mathrm{B}_{24} \mathrm{~N}_{24}$ have been optimized at B3LYP method with Def2SV(P)/ LANL2DZ(ECP) and EPR-II basis set respectively. To confirmation the structural stability of the $\mathrm{B}_{24} \mathrm{~N}_{24}-\left[\mathrm{Co}(\mathrm{CN})_{6}\right]^{3-}$ nano system, delocalization of electrons between donor and acceptor bonds and LUMO and HOMO for the lowest energy have been computed by DFT/ B3LYP method. Then we investigated NBO data such as coefficients and hybrids of orbitals, second order perturbation theory analysis of fock matrix, and " $E$ in different loops of the nanoring have been calculated at B3LYP method.
\end{abstract}

Key words: DFT, ECP, EPR-II basis set, HOMO, LUMO, NICS.

\section{INTRODUCTION}

There has been a noticeable regard in experimental researches of $B_{n} N_{m}$ nanoring. $B_{n} N_{n}$ have been provided by reaction of $\mathrm{BCl}_{3}$ with $\mathrm{NH}_{3}$ in a laser beam ${ }^{1,2}$. The experimental data of synthesis and various spectrometers are requirement to guess structural stabilities and consider physical chemistry properties of such molecules of $\mathrm{B}_{24} \mathrm{C}_{12} \mathrm{~N}_{24}$ molecule ${ }^{3}$ and the $\mathrm{B}_{12} \mathrm{~N}_{12}, \mathrm{~B}_{16} \mathrm{~N}_{16}$ and $\mathrm{B}_{28} \mathrm{~N}_{28}$ molecules $^{4-6}$. In present work we have utilize single wall $\mathrm{B}_{24} \mathrm{~N}_{24}$ borane nitride nanoring. The schematic of $\mathrm{B}_{24} \mathrm{~N}_{24}$ is displayed in the Figure 1. After valence bond theory was corroborated, Molecular orbital theory had been prospered. Thus, we presentation the non-bonded interaction of the $\left[\mathrm{Co}(\mathrm{CN})_{6}\right]^{3-}$ Situated $\mathrm{B}_{24} \mathrm{~N}_{24}$ nano ring. The basically purpose of this investigation was the study of the electromagnetic interactions within the $\mathrm{B}_{24} \mathrm{~N}_{24}$ $\left[\mathrm{Co}(\mathrm{CN})_{6}\right]^{3-}$ system. For further evaluation about electromagnetic interactions, stability structure of $\left[\mathrm{Co}(\mathrm{CN})_{6}\right]^{3-}$ complex affected various loops of nano ring have been computed. For further structural data, bond gaps and the hybrids on atom have been reported to estimate the structural ability of the $\left[\mathrm{Co}(\mathrm{CN})_{6}\right]^{3-}$ to make a stable $\mathrm{B}_{24} \mathrm{~N}_{24}-\left[\mathrm{Co}(\mathrm{CN})_{6}\right]^{3-}$ system. 


\section{Computational details}

To determination electromagnetic interactions of the $\left[\mathrm{Co}(\mathrm{CN})_{6}\right]^{3-}$ complex inclusive $\mathrm{Co}$ (III) and six $\Pi$-acceptor ligands, the geometry of the $\left[\mathrm{Co}(\mathrm{CN})_{6}\right]^{3-}$ was optimized at DFT/B3LYP method with Def2-SV(P) basis set and LANL2DZ Effective Core Potential. Also, the geometry of the mix of $\mathrm{B}_{24} \mathrm{~N}_{24}$ nanoring and $\left[\mathrm{Co}(\mathrm{CN})_{6}\right]^{3-}$ complex was optimized at DFT/B3LYP method with EPR-II basis set. Thermochemical properties were determined at B3LYP/EPR-II basis set to analyze the enthalpies and Gibbs free energies ${ }^{7}$. The natural bond orbital (NBO) calculations ${ }^{8,9}$ has been used to theoretical predictions the intermolecular orbital interactions in the $\left[\mathrm{Co}(\mathrm{CN})_{6}\right]^{3-}$ and $\left.\mathrm{B}_{24} \mathrm{~N}_{24}-\mathrm{Co}(\mathrm{CN})_{6}\right]^{3-10}$. So, NBO data including coefficients and hybrids of orbitals of atoms, donor-acceptor bonds and " $\mathrm{E}$ in $\left[\mathrm{Co}(\mathrm{CN})_{6}\right]^{3-}$ complex affected various loops of the $\mathrm{B}_{24} \mathrm{~N}_{24}$ nanoring have been determined.

\section{RESULTS AND DISCUTION}

To calculate the non-bonded interaction of $\mathrm{B}_{24} \mathrm{~N}_{24}-\left[\mathrm{Co}(\mathrm{CN})_{6}\right]^{3-}$ nano system, the structure of the $\mathrm{B}_{24} \mathrm{~N}_{24}$ have been optimized at B3LYP/EPR-II basis set and structure of the $\left[\mathrm{Co}(\mathrm{CN})_{6}\right]^{3-}$ complex including cyanide that known as six $\grave{A}$-acceptor ligands, have been optimized at B3LYP/Def2-SV(P) basis set and LANL2DZ ECP. The non-bonded electromagnetic interactions of the $\left[\mathrm{Co}(\mathrm{CN})_{6}\right]^{3-}$ complex Situated nanoring have been investigated at B3LYP in different loops of the $\mathrm{B}_{24} \mathrm{~N}_{24}$ nanoring.

Table 1: Optimal quantities of $\left[\mathrm{Co}(\mathrm{CN})_{6}\right]^{3-}$.

\begin{tabular}{llll}
\hline Compound & Bond ID & bond length & bond angle \\
\hline$\left[\mathrm{Co}(\mathrm{CN})_{6}\right]^{3-}$ & $\mathrm{Co}(1)-\mathrm{C}(2)$ & 1.957632 & - \\
& $\mathrm{Co}(1)-\mathrm{C}(3)$ & 1.958069 & - \\
& $\mathrm{Co}(1)-\mathrm{C}(4)$ & 1.957556 & - \\
$\mathrm{Co}(1)-\mathrm{C}(5)$ & 1.957584 & - \\
$\mathrm{Co}(1)-\mathrm{C}(6)$ & 1.957485 & - \\
$\mathrm{Co}(1)-\mathrm{C}(7)$ & 1.957629 & - \\
$\mathrm{C}(2)-\mathrm{N}(11)$ & 1.180025 & - \\
$\mathrm{C}(3)-\mathrm{N}(8)$ & 1.179911 & - \\
$\mathrm{C}(4)-\mathrm{N}(12)$ & 1.179998 & - \\
$\mathrm{C}(5)-\mathrm{N}(10)$ & 1.180055 & - \\
$\mathrm{C}(6)-\mathrm{N}(13)$ & 1.179861 & - \\
$\mathrm{C}(7)-\mathrm{N}(9)$ & 1.180024 & - \\
$\mathrm{C}(2)-\mathrm{Co}(1)-\mathrm{C}(3)$ & - & 90.02 \\
$\mathrm{C}(2)-\mathrm{Co}(1)-\mathrm{C}(4)$ & - & 90.0163 \\
$\mathrm{C}(2)-\mathrm{Co}(1)-\mathrm{C}(5)$ & - & 89.9842 \\
$\mathrm{C}(2)-\mathrm{Co}(1)-\mathrm{C}(6)$ & - & 89.9782 \\
$\mathrm{C}(2)-\mathrm{Co}(1)-\mathrm{C}(7)$ & - & 179.9811 \\
$\mathrm{C}(3)-\mathrm{Co}(1)-\mathrm{C}(4)$ & - & 89.817 \\
$\mathrm{C}(3)-\mathrm{Co}(1)-\mathrm{C}(5)$ & - & 90.1773 \\
$\mathrm{C}(3)-\mathrm{Co}(1)-\mathrm{C}(6)$ & - & 179.9773 \\
$\mathrm{C}(3)-\mathrm{Co}(1)-\mathrm{C}(7)$ & - & 89.9877 \\
$\mathrm{C}(4)-\mathrm{Co}(1)-\mathrm{C}(5)$ & - & 179.9942 \\
$\mathrm{C}(4)-\mathrm{Co}(1)-\mathrm{C}(6)$ & - & 89.9782 \\
$\mathrm{C}(4)-\mathrm{Co}(1)-\mathrm{C}(7)$ & - & 89.9665 \\
$\mathrm{C}(5)-\mathrm{Co}(1)-\mathrm{C}(6)$ & - & 90.033 \\
$\mathrm{C}(5)-\mathrm{Co}(1)-\mathrm{C}(7)$ & - & 90.014 \\
$\mathrm{C}(6)-\mathrm{Co}(1)-\mathrm{C}(7)$ & - & \\
& & \\
\hline
\end{tabular}

* See Figure 1 for more details 
Table 2: Natural atomic orbitals of $\left[\mathrm{Co}(\mathrm{CN})_{6}\right]^{3 \cdot c o m p l e x}$

\begin{tabular}{|c|c|c|c|}
\hline \multirow[t]{2}{*}{ Atom } & \multirow[b]{2}{*}{$\begin{array}{l}\text { Atomic } \\
\text { Orbital }\end{array}$} & \multicolumn{2}{|c|}{$\begin{array}{l}\text { Natural atomic orbital } \\
\qquad\left[\mathrm{CoF}_{6}\right]^{-3-}\end{array}$} \\
\hline & & Occupancy & Energy \\
\hline \multirow[t]{6}{*}{$\mathrm{Co}^{3+}(1)$} & $4 \mathrm{~s}$ & 0.46228 & 2.90416 \\
\hline & $3 d y z$ & 1.84859 & 0.10792 \\
\hline & $3 d x y$ & 1.73218 & 0.11879 \\
\hline & $3 d z 2$ & 1.68785 & 0.12323 \\
\hline & $3 d x 2 y 2$ & 1.41028 & 0.14943 \\
\hline & $3 d x z$ & 1.06674 & 0.18220 \\
\hline \multirow[t]{4}{*}{ C (2) } & $2 s$ & 1.25718 & 0.22394 \\
\hline & $2 p x$ & 0.82429 & 0.33754 \\
\hline & $2 p y$ & 0.98223 & 0.48570 \\
\hline & $2 p z$ & 0.81132 & 0.32525 \\
\hline \multirow[t]{4}{*}{ C (3) } & $2 s$ & 1.25730 & 0.22404 \\
\hline & $2 p x$ & 0.89856 & 0.40722 \\
\hline & $2 p y$ & 0.81923 & 0.33271 \\
\hline & $2 p z$ & 0.89995 & 0.40823 \\
\hline \multirow[t]{4}{*}{ C (4) } & $2 s$ & 1.25716 & 0.22399 \\
\hline & $2 p x$ & 0.89507 & 0.40400 \\
\hline & $2 p y$ & 0.81626 & 0.32990 \\
\hline & $2 p z$ & 0.90656 & 0.41453 \\
\hline \multirow[t]{4}{*}{ C (5) } & $2 s$ & 1.25716 & 0.22389 \\
\hline & $2 p x$ & 0.89526 & 0.40429 \\
\hline & $2 p y$ & 0.81626 & 0.32996 \\
\hline & $2 p z$ & 0.90628 & 0.41436 \\
\hline \multirow[t]{4}{*}{ C (6) } & $2 s$ & 1.25697 & 0.22387 \\
\hline & $2 p x$ & 0.89884 & 0.40752 \\
\hline & $2 p y$ & 0.81930 & 0.33276 \\
\hline & $2 p z$ & 0.89969 & 0.40810 \\
\hline \multirow[t]{4}{*}{ C (7) } & $2 s$ & 1.25717 & 0.22396 \\
\hline & $2 p x$ & 0.82428 & 0.33752 \\
\hline & $2 p y$ & 0.98225 & 0.48576 \\
\hline & $2 \mathrm{pz}$ & 0.81131 & 0.32525 \\
\hline \multirow[t]{4}{*}{$N(8)$} & $2 s$ & 1.58723 & -0.18862 \\
\hline & $2 p x$ & 1.40270 & 0.20756 \\
\hline & 2py & 1.24637 & 0.24158 \\
\hline & $2 p z$ & 1.40643 & 0.20663 \\
\hline \multirow[t]{4}{*}{$N(9)$} & $2 s$ & 1.58725 & -0.18838 \\
\hline & $2 p x$ & 1.25623 & 0.23963 \\
\hline & $2 p y$ & 1.56854 & 0.17157 \\
\hline & $2 p z$ & 1.23059 & 0.24522 \\
\hline \multirow[t]{4}{*}{$N(10)$} & $2 s$ & 1.58726 & -0.18837 \\
\hline & $2 p x$ & 1.39603 & 0.20922 \\
\hline & $2 p y$ & 1.24036 & 0.24311 \\
\hline & $2 p z$ & 1.41899 & 0.20415 \\
\hline
\end{tabular}

\begin{tabular}{llll}
$\mathrm{N}(11)$ & $2 \mathrm{~s}$ & 1.58725 & -0.18841 \\
& $2 \mathrm{px}$ & 1.25623 & 0.23960 \\
& $2 \mathrm{py}$ & 1.56853 & 0.17156 \\
$\mathrm{~N}(12)$ & $2 \mathrm{pz}$ & 1.23061 & 0.24519 \\
& $2 \mathrm{~s}$ & 1.58724 & -0.18845 \\
& $2 \mathrm{px}$ & 1.39563 & 0.20925 \\
& $2 \mathrm{py}$ & 1.24032 & 0.24305 \\
& $2 \mathrm{pz}$ & 1.41942 & 0.20395 \\
$\mathrm{~N}(13)$ & $2 \mathrm{~s}$ & 1.58716 & -0.18836 \\
& $2 \mathrm{px}$ & 1.40311 & 0.20764 \\
& $2 \mathrm{py}$ & 1.24641 & 0.24176 \\
& $2 \mathrm{pz}$ & 1.40595 & 0.20693 \\
\hline
\end{tabular}

Optimized parameters of $\left[\mathrm{Co}(\mathrm{CN})_{6}\right]^{3-}$ consists bond lengths and bond angles have been reported in Table 1. We can get all of the Co-C bond lengths are the same in values and all of the $\mathrm{C}-\mathrm{N}$ bond lengths are same to other, because the octahedral compounds that are Low-spin $d^{6}$ electronic configuration such as Co (III) with six П-acceptor ligands no indicate the Jahn-Teller distortion. But the octahedral compounds that are High-spin $d^{6}$ electronic configuration such as Co (III) with six $\sigma$ donor ligands indicate the Jahn-Teller distortion ${ }^{11}$. Pursuant the occupancy and energy values of Co (III) metal in Table 2, we can get that $3 d_{y z}, 3 d_{x y}$, $3 d_{z 2}$ orbitals include two electrons and the least value of energy. The other $d$ orbitals and $4 \mathrm{~s}$ orbital include no electron. Also, Pursuant the occupancy and energy values of 6 - -acceptor ligands it was demonstrated that $2 \mathrm{~s}$ orbital of $\mathrm{N}$ atom participate to creation the $\sigma$ molecular orbitals and one non bonding electron pairs of $\mathrm{N}$ situate in $2 \mathrm{p}$ orbital that has higher energy level. For instance, in $\mathrm{N}(8)$ and $N(13)$ that are in same position relative to the Co (III), $2 p_{y}$ has a higher energy levels and $2 p_{x}$ and $2 p_{z}$ have lower energy levels. In addition, pursuant Table 2 data, atom pairs $\mathrm{N}(8)-\mathrm{N}(13), \mathrm{N}(9)-\mathrm{N}(11), \mathrm{N}(10)$ $\mathrm{N}(12)$ in $\left[\mathrm{Co}(\mathrm{CN})_{6}\right]^{3-}$ complex that are in same position relative to $\mathrm{Co}$ (III), have the same occupancy and energy levels (Fig. 1). The energy level difference of metal-ligands bonding in $\left[\mathrm{Co}(\mathrm{CN})_{6}\right]^{3-}$ complex have been reported in Table 3. We can understand, the size of " field strength. (CN)- ligand is a strong field ligand that increase " omore than F- ligand as weak field ligand. Determination of second order perturbation theory analysis of fock matrix of $\mathrm{C}$ and $\mathrm{N}$ atoms at the level of B3LYP/EPR-II basis set and Co (III) atom at the level of B3LYP/ Def2-SV(P)/LANL2DZ (ECP) 
Table 3: Molecular orbital diagram of $\left[\mathrm{Co}(\mathrm{CN})_{6}\right]^{3-c o m p l e x .}$

\begin{tabular}{|c|c|c|c|c|}
\hline \multirow[t]{2}{*}{ Compound } & \multicolumn{4}{|c|}{ Molecular orbital diagram } \\
\hline & \multicolumn{2}{|c|}{ Natural Bond Orbitals } & Occupancy & Energy (a.u.) \\
\hline \multirow[t]{16}{*}[\mathrm{Co}(\mathrm{CN})_{6}]{$^{3-}$} & $\mathrm{BD}^{*}(1) \mathrm{Co} 1-\mathrm{C} 6$ & $\Pi_{t 1 u}^{*}$ & 0.43225 & 1.40362 \\
\hline & $\mathrm{BD}^{\star}(1) \mathrm{Co} 1-\mathrm{C} 5$ & $\sigma^{*}$ & 0.43283 & 1.40319 \\
\hline & $\mathrm{BD}^{*}(3) \mathrm{C} 5-\mathrm{N} 10$ & n.b. & 0.05113 & 0.50551 \\
\hline & $\mathrm{BD}^{*}(3) \mathrm{C} 6-\mathrm{N} 13$ & n.b. & 0.05112 & 0.50548 \\
\hline & $\mathrm{BD}^{\star}(3) \mathrm{C} 7-\mathrm{N} 9$ & n.b. & 0.05105 & 0.50547 \\
\hline & $\mathrm{BD}^{*}(3) \mathrm{C} 2-\mathrm{N} 11$ & n.b. & 0.05105 & 0.50546 \\
\hline & $\mathrm{BD}^{\star}(3) \mathrm{C} 3-\mathrm{N} 8$ & n.b. & 0.05107 & 0.50544 \\
\hline & $\mathrm{BD}^{*}(3) \mathrm{C} 4-\mathrm{N} 12$ & n.b. & 0.05113 & 0.50543 \\
\hline & LP (1) C 3 & $\sigma^{*}$ & 1.50469 & 0.35364 \\
\hline & $\operatorname{LP}(1) \mathrm{C} 7$ & $\Pi_{\mathrm{t} 1 \mathrm{u}}^{\mathrm{arg}}$ & 1.50443 & 0.35360 \\
\hline & LP ( 1) C 4 & $\sigma_{\text {eq }}^{*}$ & 1.50444 & 0.35359 \\
\hline & LP ( 1)Co 1 & $\Pi_{\mathrm{t} 2 \mathrm{~g}}$ & 1.91544 & 0.10051 \\
\hline & $\mathrm{BD}$ ( 1)Co 1- C 5 & $\sigma_{\mathrm{eq}}$ & 1.91189 & -0.05029 \\
\hline & $\mathrm{BD}$ ( 1)Co 1- C 2 & $\sigma_{\mathrm{t} 1 \mathrm{u}}$ & 1.91190 & -0.05030 \\
\hline & BD ( 1)Co 1- C 6 & $\sigma_{\text {a1g }}$ & 1.91188 & -0.05039 \\
\hline & $\Delta_{\text {oct }}$ & & 0.25308 a.u. & \\
\hline
\end{tabular}

Table 4: Natural bond orbital (NBO) analysis of $\left[\mathrm{Co}(\mathrm{CN})_{6}\right]^{3-}$

\begin{tabular}{|c|c|c|c|c|c|}
\hline \multirow{2}{*}{$\begin{array}{l}\text { Compound } \\
{\left[\mathrm{Co}(\mathrm{CN})_{6}\right]^{3-}}\end{array}$} & \multicolumn{5}{|c|}{ Natural bond orbital (NBO) analysis } \\
\hline & Donor NBO (i) & Acceptor NBO (j) & $\mathrm{E}(2) \mathrm{kcal} / \mathrm{mol}$ & $E(j)-E(i) a . u$. & F(i,j)a.u. \\
\hline & $\mathrm{BD}(1) \mathrm{Co} 1-\mathrm{C} 2$ & $\mathrm{BD}^{*}(1) \mathrm{Co} 1-\mathrm{C} 5$ & 1.65 & 1.45 & 0.048 \\
\hline & $\mathrm{BD}$ ( 1)Co 1- C 2 & $\mathrm{BD}^{*}(1) \mathrm{Co} 1-\mathrm{C} 6$ & 1.65 & 1.45 & 0.048 \\
\hline & $\mathrm{BD}$ ( 1)Co 1- C 2 & $\mathrm{BD}^{*}(3) \mathrm{C} 6-\mathrm{N} 13$ & 2.23 & 0.56 & 0.032 \\
\hline & $\mathrm{BD}$ ( 1)Co 1- C 5 & $\mathrm{BD}^{*}(1)$ Co $1-\mathrm{C} 6$ & 1.66 & 1.45 & 0.048 \\
\hline & $\mathrm{BD}$ ( 1)Co 1- C 5 & $\mathrm{BD}^{*}(3) \mathrm{C} 2-\mathrm{N} 11$ & 1.24 & 0.56 & 0.024 \\
\hline & $\mathrm{BD}$ ( 1)Co 1- C 6 & $\mathrm{BD}^{*}(1)$ Co $1-\mathrm{C} 5$ & 1.67 & 1.45 & 0.048 \\
\hline & $\mathrm{BD}$ ( 1)Co 1- C 6 & $\mathrm{BD}^{*}(3) \mathrm{C} 2-\mathrm{N} 11$ & 1.06 & 0.56 & 0.022 \\
\hline & $\mathrm{BD}$ ( 1)Co 1- C 6 & $\mathrm{BD}^{*}(3) \mathrm{C} 5-\mathrm{N} 10$ & 2.27 & 0.56 & 0.032 \\
\hline & $\mathrm{BD}$ ( 2) C 6- N13 & $\mathrm{BD}^{*}(3) \mathrm{C} 5-\mathrm{N} 10$ & 0.6 & 0.43 & 0.014 \\
\hline & $\operatorname{LP}(1) \operatorname{Co} 1$ & $\mathrm{BD}^{*}(3) \mathrm{C} 6-\mathrm{N} 13$ & 2.04 & 0.4 & 0.026 \\
\hline & $\mathrm{BD}$ ( 1)Co 1- C 5 & $\mathrm{BD}^{*}(3) \mathrm{C} 3-\mathrm{N} 8$ & 2.25 & 0.56 & 0.032 \\
\hline & $\operatorname{LP}(1) \operatorname{Co} 1$ & $\mathrm{BD}^{*}(3) \mathrm{C} 3-\mathrm{N} 8$ & 0.13 & 0.4 & 0.007 \\
\hline & $\mathrm{BD}$ ( 1)Co 1- C 2 & $\mathrm{BD}^{*}(3) \mathrm{C} 4-\mathrm{N} 12$ & 2.29 & 0.56 & 0.032 \\
\hline & $\operatorname{LP}(1)$ Co 1 & $\mathrm{BD}^{*}$ ( 3) C 4- N12 & 1.59 & 0.4 & 0.023 \\
\hline & $\mathrm{BD}$ ( 1)Co 1- C 5 & $\mathrm{BD}^{*}($ 3) C 7- N 9 & 1.3 & 0.56 & 0.024 \\
\hline & $\mathrm{BD}$ ( 1)Co 1- C 6 & $\mathrm{BD}^{*}$ ( 3) C 7- N 9 & 1.02 & 0.56 & 0.021 \\
\hline & $\operatorname{LP}(1)$ Co 1 & $\mathrm{BD}^{*}(2) \mathrm{C} 7-\mathrm{N} 9$ & 3.73 & 0.4 & 0.035 \\
\hline & LP (1) C 3 & BD*(1)Co 1- C 5 & 345.09 & 1.05 & 0.546 \\
\hline & $\operatorname{LP}(1) \mathrm{C} 3$ & $\mathrm{BD}^{*}(1)$ Co $1-\mathrm{C} 6$ & 585.34 & 1.05 & 0.711 \\
\hline & $\operatorname{LP}(1) \mathrm{C} 3$ & $\mathrm{BD}^{*}(3) \mathrm{C} 2-\mathrm{N} 11$ & 4.15 & 0.15 & 0.025 \\
\hline & $\operatorname{LP}(1) \mathrm{C} 3$ & $\mathrm{BD}^{*}(3) \mathrm{C} 5-\mathrm{N} 10$ & 10.16 & 0.15 & 0.04 \\
\hline & LP ( 1) C 3 & $\mathrm{BD}^{*}$ (3) C 7- N 9 & 3.82 & 0.15 & 0.024 \\
\hline
\end{tabular}


have been shown in Table 4. Also Bond orbital, Coefficients and Hybrids of $\left[\mathrm{Co}(\mathrm{CN})_{6}\right]^{3-}$ complex have been shown in Table 5 . In accordance with data of Table 5, we can get that the bonding and anti-bonding coefficients of orbitals of Co-C and $\mathrm{Co}-\mathrm{N}$ bonds were 0.8 and 0.7 respectively. To calculation non-bonded interaction of the $\left[\mathrm{Co}(\mathrm{CN})_{6}\right]^{3-}$ complex Situated in nano ring field, we attend on the $\mathrm{B}_{24} \mathrm{~N}_{24}$ nano ring and optimized structure of the $\mathrm{B}_{24} \mathrm{~N}_{24}-\left[\mathrm{Co}(\mathrm{CN})_{6}\right]^{3-}$ system have been displayed in Fig.1. The geometry of $\mathrm{B}_{24} \mathrm{~N}_{24}$ nano ring has been optimized at B3LYP method with EPR-II basis set. According to the frequency calculation for $\mathrm{B}_{24} \mathrm{~N}_{24}$ nano rings, thermochemical quantities were equal to $\Delta \mathrm{G}=-97.6323205765 \mathrm{kcal} /$ $\mathrm{mol}$ and $\Delta \mathrm{H}=-166.384143925 \mathrm{kcal} / \mathrm{mol}$, corroborated the structural stability of nano rings. Dipole moments of alone complex and complex affected various loops of nanoring have been shown in Table 6. The geometry of mix of $\mathrm{B}_{24} \mathrm{~N}_{24}$ and $\left[\mathrm{Co}(\mathrm{CN})_{6}\right]^{3-}$ complex have been optimized at B3LYP method with EPR-II basis set for B,N,C atoms and

Table 5: Bond orbital, Coefficients, Hybrids of $\left[\mathrm{Co}(\mathrm{CN})_{6}\right]^{3-}$ at NBO studies

\begin{tabular}{|c|c|c|}
\hline \multirow[t]{2}{*}{ Compound } & \multicolumn{2}{|c|}{ Natural bond orbital (NBO) analysis } \\
\hline & Bond orbital & Coefficients/ Hybrids \\
\hline \multirow{13}{*}[\mathrm{Co}(\mathrm{C})_{6}]{$^{3-}$} & $\mathrm{BD}$ ( 1)Co 1- C 2 & $0.5127^{\star} \mathrm{Co} 1\left(\mathrm{sp}{ }^{0.00} \mathrm{~d}^{2.00}\right)+0.8586^{\star} \mathrm{C} 2\left(\mathrm{sp}{ }^{0.79} \mathrm{~d}^{0.00}\right)$ \\
\hline & $\mathrm{BD}$ ( 1)Co 1- C 5 & $0.5127^{\star} \operatorname{Co~} 1\left(\mathrm{sp}^{0.00} \mathrm{~d}^{2.00}\right)+0.8585^{\star} \mathrm{C} 5\left(\mathrm{sp}^{0.79} \mathrm{~d}^{0.00}\right)$ \\
\hline & $\mathrm{BD}$ ( 1)Co 1- C 6 & $0.5128^{\star} \mathrm{Co} 1\left(\mathrm{sp}^{0.00} \mathrm{~d}^{2.00}\right)+0.8585^{\star} \mathrm{C} 6\left(\mathrm{sp}{ }^{0.79} \mathrm{~d}^{0.00}\right)$ \\
\hline & LP ( 1)Co 1 & $\left(s p{ }^{0.00} d^{1.00}\right)$ \\
\hline & LP ( 1) C 3 & $\left(s p{ }^{1.10} d^{0.00}\right)$ \\
\hline & $\mathrm{BD}^{*}(1) \mathrm{Co} 1-\mathrm{C} 5$ & $0.8585^{\star}$ Co $1\left(s p{ }^{0.00} d^{2.00}\right)-0.5127^{\star} \mathrm{C} 5\left(s p{ }^{0.79} d^{0.00}\right)$ \\
\hline & $B D^{*}(1)$ Co $1-C 6$ & $0.8585^{\star} \mathrm{Co} 1\left(\mathrm{sp}{ }^{0.00} \mathrm{~d}^{2.00}\right)-0.5128^{\star} \mathrm{C} 6\left(\mathrm{sp}{ }^{0.79} \mathrm{~d}^{0.00}\right)$ \\
\hline & $\mathrm{BD}^{*}(3) \mathrm{C} 2-\mathrm{N} 11$ & $0.7800^{\star} \mathrm{C} 2\left(\mathrm{sp}^{1.00} \mathrm{~d}^{0.00}\right)-0.6258^{\star} \mathrm{N} 11\left(\mathrm{sp}^{1.00} \mathrm{~d}^{0.00}\right)$ \\
\hline & $\mathrm{BD}^{*}(3) \mathrm{C} 3-\mathrm{N} 8$ & $0.7800^{*} \mathrm{C} 3\left(\mathrm{sp}^{1.00} \mathrm{~d}^{0.00}\right)-0.6258^{*} \mathrm{~N} 8\left(\mathrm{sp}^{1.00} \mathrm{~d}^{0.00}\right)$ \\
\hline & $\mathrm{BD}^{*}(3) \mathrm{C} 4-\mathrm{N} 12$ & $0.7800^{*} \mathrm{C} 4\left(\mathrm{sp}{ }^{1.00} \mathrm{~d}^{0.00}\right)-0.6258^{\star} \mathrm{N} 12\left(\mathrm{sp}{ }^{1.00} \mathrm{~d}^{0.00}\right)$ \\
\hline & $\mathrm{BD}^{*}(3) \mathrm{C} 5-\mathrm{N} 10$ & $0.7800^{\star} \mathrm{C} 5\left(\mathrm{sp}^{1.00} \mathrm{~d}^{0.00}\right)-0.6258^{\star} \mathrm{N} 10\left(\mathrm{sp}{ }^{1.00} \mathrm{~d}^{0.00}\right)$ \\
\hline & $\mathrm{BD}^{*}(3) \mathrm{C} 6-\mathrm{N} 13$ & $0.7800^{\star} \mathrm{C} 6\left(\mathrm{sp}^{1.00} \mathrm{~d}^{0.00}\right)-0.6258^{\star} \mathrm{N} 13\left(\mathrm{sp}{ }^{1.00} \mathrm{~d}^{0.00}\right)$ \\
\hline & $\mathrm{BD}^{*}(3) \mathrm{C} 7-\mathrm{N} 9$ & $0.7800^{*} \mathrm{C} 7\left(\mathrm{sp}^{1.00} \mathrm{~d}^{0.00}\right)-0.6258^{*} \mathrm{~N} 9\left(\mathrm{sp}^{1.00} \mathrm{~d}^{0.00}\right)$ \\
\hline
\end{tabular}

Table 6: Changes in the relative energies $(\Delta E)$, dipole moment $(r)$, nuclear repulsion energy and bond gap of alone $\left[\mathrm{Co}(\mathrm{CN})_{6}\right]^{3-}$ and affected various loops of $\mathrm{B}_{24} \mathrm{~N}_{24}$

\begin{tabular}{|c|c|c|c|c|c|}
\hline \multirow[t]{2}{*}{$\begin{array}{l}\text { Compound } \\
\mathrm{B}_{24} \mathrm{~N}_{24}-\left[\mathrm{Co}(\mathrm{CN})_{6}\right]^{3-}\end{array}$} & \multicolumn{5}{|c|}{$\begin{array}{r}\text { Basis sets for } \mathrm{Co}^{3+} \\
\text { Def2-SV(P), LANL2DZ ECP }\end{array}$} \\
\hline & $\begin{array}{l}\text { band gap } \\
\text { (Hartree) }\end{array}$ & $\begin{array}{c}\Delta \mathrm{E} \\
\text { (Hartree) }\end{array}$ & $\begin{array}{c}\text { Dipole } \\
\text { moment (Debye) }\end{array}$ & NICS & $\begin{array}{l}\text { nuclear repulsion } \\
\text { energy (Hartree) }\end{array}$ \\
\hline$\left[\mathrm{Co}(\mathrm{CN})_{6}\right]^{3-}$ & 0.25308 & -701.17098 & 0.0017 & * & 748.95533 \\
\hline loop 1-[Co(CN $\left.)_{6}\right]^{3-}$ & 0.02448 & -940.01508 & 4.9114 & -10.1058 & 1219.24152 \\
\hline loop 2-[Co(CN $\left.)_{6}\right]^{3-}$ & 0.0329 & -940.03334 & 5.3568 & -10.1189 & 1212.20610 \\
\hline loop 3-[Co $\left.(\mathrm{CN})_{6}\right]^{3-}$ & 0.0237 & -940.01497 & 5.0787 & -10.1058 & 1219.24129 \\
\hline loop 4-[Co(CN $\left.)_{6}\right]^{3-}$ & 0.03195 & -940.03336 & 5.3016 & -10.1189 & 1212.20822 \\
\hline loop 5-[Co(CN $\left.)_{6}\right]^{3-}$ & 0.03372 & -940.01429 & 5.5595 & -10.1058 & 1219.25988 \\
\hline loop 6-[Co(CN) $\left.)_{6}\right]^{3-}$ & 0.03481 & -940.03316 & 5.5523 & -10.1189 & 1212.18374 \\
\hline loop 7-[Co(CN $\left.)_{6}\right]^{3-}$ & 0.02262 & -940.01477 & 4.6646 & -10.1058 & 1219.25565 \\
\hline loop 8-[Co(CN $\left.)_{6}\right]^{3-}$ & 0.0313 & -940.03326 & 5.3537 & -10.1189 & 1212.18442 \\
\hline
\end{tabular}




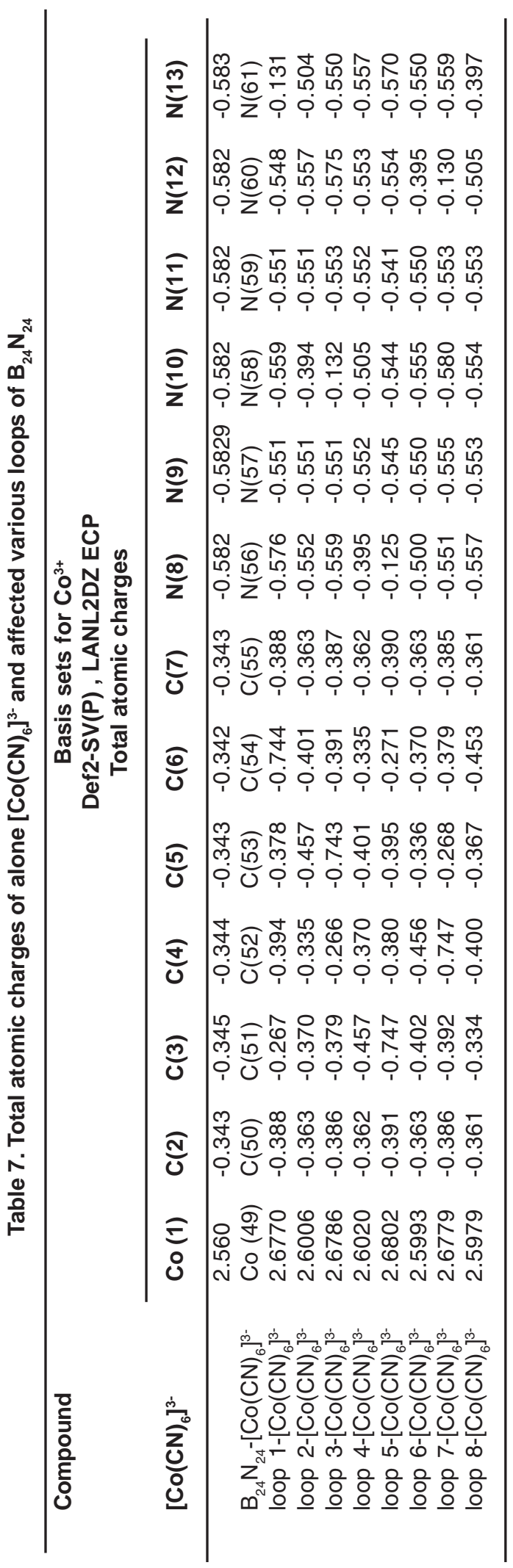

Def2-SV $(P)$ basis set and LANL2DZ ECP for Co (III). According to the electron paramagnetic resonance (EPR) calculate, it is noteworthy that the energy obtained from the mentioned basis set and $\mathrm{ECP}$ for alone $\mathrm{B}_{24} \mathrm{~N}_{24}$ nanoring and alone $\left[\mathrm{Co}(\mathrm{CN})_{6}\right]^{3-}$ complex were equal to -1911.727563 and 701.1710148 (Hartree) respectively. To describes the non-bonded interaction of $\left[\mathrm{Co}(\mathrm{CN})_{6}\right]^{3-}$ affected eight various loops of $\mathrm{B}_{24} \mathrm{~N}_{24}$ nano ring, we focus on quantities values such as the relative energies ("E), dipole moment (r), nuclear repulsion energy, NICS and bond gap that mentioned values have been displayed in Table 6. Atomic charge is the physical property of matter that causes it to experience a force when close to other electrically charged matter. So, total atomic charge of alone $\left[\mathrm{Co}(\mathrm{CN})_{6}\right]^{3-}$ complex atoms and under different loops of $\mathrm{B}_{24} \mathrm{~N}_{24}$ nanoring, have been reported in Table 7. Also, Bond Length, Total atomic charges and Dipole orientation of atoms of different loops of nanorings have been displayed in Table 8. Magnetic Resonance parameters of $\left[\mathrm{Co}(\mathrm{CN})_{6}\right]^{3-}$ complexes under three CSGT, GIAO, IGAIM methods have been shown in Table 9.
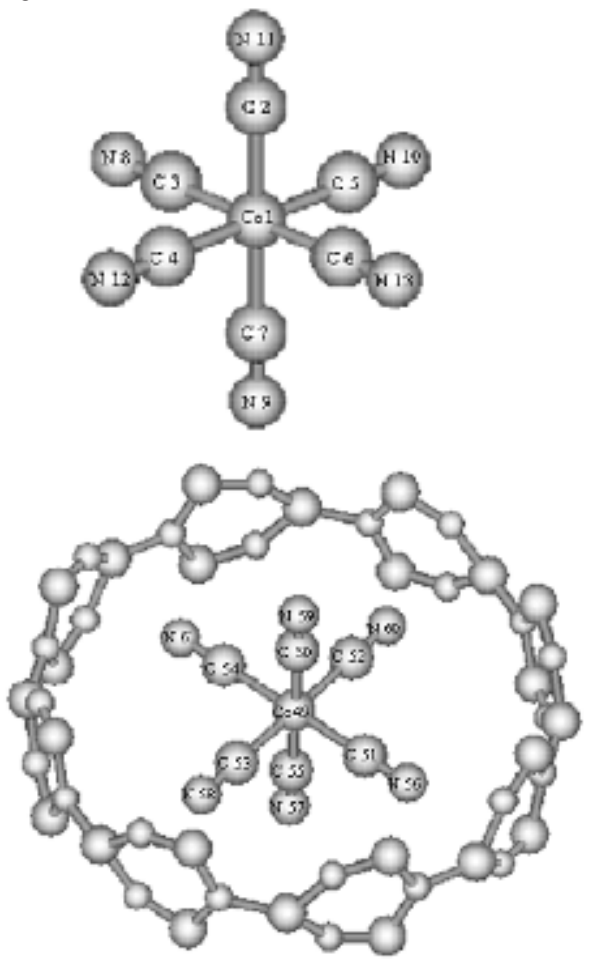

Fig. 1: Optimal structure of $\left[\mathrm{Co}(\mathrm{CN})_{6}\right]^{3-}$ stand alone and situated $\mathrm{B}_{24} \mathrm{~N}_{24}$ nanoring 
Table 8: Bond Length, Atomic charges \& Dipole orientation of the sides of various loops of $B_{24} N_{24}$.

\begin{tabular}{|c|c|c|c|c|c|}
\hline \multirow[t]{2}{*}{$\begin{array}{l}\text { Compound } \\
\left.\mathrm{B}_{24} \mathrm{~N}_{24}-\mathrm{Co}(\mathrm{CN})_{6}\right]^{3-}\end{array}$} & \multirow{2}{*}{\multicolumn{5}{|c|}{$\begin{array}{c}\text { Basis sets of Co (III) } \\
\text { Def2-SV(P), LANL2DZ ECP }\end{array}$}} \\
\hline & & & & & \\
\hline \multirow[t]{6}{*}{ loop 1} & $\mathrm{~B}(1)$ & $r_{1-2}$ & 1.303 & 0.029792 & 90.0174 .2630 \\
\hline & $N(2)$ & $r_{1-32}^{r-2}$ & 1.417 & -0.059818 & \\
\hline & $\mathrm{B}(3)$ & $r_{2-3}^{1-32}$ & 1.466 & 0.030860 & \\
\hline & $\mathrm{N}(32)$ & $r_{3-34}^{2-3}$ & 1.466 & -0.166730 & \\
\hline & $\mathrm{B}(33)$ & $r_{32-33}^{3-34}$ & 1.417 & 0.029797 & \\
\hline & $\mathrm{N}(34)$ & $r_{33-34}^{32-33}$ & 1.303 & -0.059851 & \\
\hline \multirow[t]{6}{*}{ loop 2} & $N(4)^{\prime}$ & $r_{4-5}^{33-34}$ & 1.417 & -0.143033 & 90.0147 .1122 \\
\hline & $\mathrm{B}(5)$ & $r_{4-35}^{4-5}$ & 1.417 & -0.003640 & \\
\hline & $N(6)$ & $r_{5-6}^{4-30}$ & 1.303 & -0.036094 & \\
\hline & $\mathrm{B}(7)$ & $r_{6-7}^{5-6}$ & 1.466 & 0.025729 & \\
\hline & $\mathrm{B}(35)$ & $r_{7-36}^{6-1}$ & 1.466 & -0.003627 & \\
\hline & $N(36)$ & $r_{35-36}^{1-36}$ & 1.303 & -0.036107 & \\
\hline \multirow[t]{6}{*}{ loop 3} & $N(8)^{\prime}$ & $r_{8-9}^{35-30}$ & 1.417 & -0.166571 & 90.094 .6723 \\
\hline & $\mathrm{B}(9)$ & $r_{8-37}^{8-9}$ & 1.417 & 0.028774 & \\
\hline & $N(10)$ & $r_{9-10}^{8-37}$ & 1.303 & -0.058331 & \\
\hline & $B(11)$ & $r_{10-11}^{9-10}$ & 1.466 & 0.023939 & \\
\hline & $\mathrm{B}(37)$ & $r_{11-38}^{0-11}$ & 1.466 & 0.028554 & \\
\hline & $N(38)$ & $r_{37-38}$ & 1.303 & -0.058119 & \\
\hline \multirow[t]{6}{*}{ loop 4} & $\mathrm{~N}(12)$ & $r_{12-13}^{3 /-38}$ & 1.417 & -0.141968 & 90.057 .3710 \\
\hline & $\mathrm{B}(13)$ & $r_{12-39}^{12-73}$ & 1.417 & -0.003813 & \\
\hline & $N(14)$ & $r_{13-14}$ & 1.303 & -0.035563 & \\
\hline & $\mathrm{B}(15)$ & $r_{14-15}^{13-14}$ & 1.466 & 0.026477 & \\
\hline & $\mathrm{B}(39)$ & $r_{15-40}^{14-10}$ & 1.466 & -0.003821 & \\
\hline & $N(40)$ & $r_{39-40}$ & 1.303 & -0.035568 & \\
\hline \multirow[t]{6}{*}{ loop 5} & $N(16)$ & $r_{16-17}$ & 1.417 & -0.176228 & 90.03 .9639 \\
\hline & $\mathrm{B}(17)$ & $r_{16-41}^{10-1 / 7}$ & 1.417 & 0.027328 & \\
\hline & $N(18)$ & $r_{17-18}$ & 1.303 & -0.066071 & \\
\hline & $\mathrm{B}(19)$ & $r_{18-19}$ & 1.466 & 0.025758 & \\
\hline & $\mathrm{B}(41)$ & $r_{19-42}^{18-19}$ & 1.466 & 0.028672 & \\
\hline & $N(42)$ & $r_{41-42}^{19-42}$ & 1.303 & -0.061409 & \\
\hline \multirow[t]{6}{*}{ Ioop 6} & $N(20)$ & $r_{20-21}^{41-4 c}$ & 1.417 & -0.145945 & 90.033 .7029 \\
\hline & $\mathrm{B}(21)$ & $r_{20-43}^{20-2 T}$ & 1.417 & -0.004382 & \\
\hline & $\mathrm{N}(22)$ & $r_{21-22}^{20-43}$ & 1.303 & -0.038261 & \\
\hline & $\mathrm{B}(23)$ & $r_{22-23}^{21-22}$ & 1.466 & 0.027356 & \\
\hline & $\mathrm{B}(43)$ & $r_{23-44}^{2<-20}$ & 1.466 & -0.004393 & \\
\hline & $\mathrm{N}(44)$ & $r_{43-44}^{23-44}$ & 1.303 & -0.038252 & \\
\hline \multirow[t]{6}{*}{ Ioop 7} & $\mathrm{~N}(24)$ & $r_{24-25}^{43-44}$ & 1.417 & -0.167045 & 90.082 .9711 \\
\hline & $\mathrm{B}(25)$ & $r_{24-45}^{24-\angle 0}$ & 1.417 & 0.032748 & \\
\hline & $\mathrm{N}(26)$ & $r_{25-26}^{24-40}$ & 1.303 & -0.057311 & \\
\hline & $\mathrm{B}(27)$ & $r_{26-27}^{20-20}$ & 1.466 & 0.030136 & \\
\hline & $\mathrm{B}(45)$ & $r_{27-46}^{20-\angle r}$ & 1.466 & 0.034124 & \\
\hline & $N(46)$ & $r_{45-46}^{27-46}$ & 1.303 & -0.059718 & \\
\hline \multirow[t]{6}{*}{ Ioop 8} & $\mathrm{~N}(28)$ & $r_{28-29}^{45-46}$ & 1.417 & -0.141699 & 90.0122 .8949 \\
\hline & $\mathrm{B}(29)$ & $r_{28-47}^{28-29}$ & 1.417 & -0.004621 & \\
\hline & $N(30)$ & $r_{29-30}^{20-4 r}$ & 1.303 & -0.035262 & \\
\hline & $\mathrm{B}(31)$ & $r_{30-31}^{25-00}$ & 1.466 & 0.025256 & \\
\hline & $\mathrm{B}(47)$ & $r_{31-48}^{30-51}$ & 1.466 & -0.004614 & \\
\hline & $N(48)$ & $r_{47-48}^{01-40}$ & 1.303 & -0.035254 & \\
\hline
\end{tabular}


Table 9: Nuclear Magnetic Resonance parameters of $\left[\mathrm{Co}(\mathrm{CN})_{6}\right]^{3-}$ complex at three CSGT,GIAO,IGAIM methods

\begin{tabular}{|c|c|c|c|c|c|c|c|}
\hline \multirow{2}{*}{$\begin{array}{l}\text { Compound } \\
{\left[\mathrm{Co}(\mathrm{CN})_{6}\right]^{3-}}\end{array}$} & \multicolumn{7}{|c|}{ NMR parameters (ppm) } \\
\hline & Isotropic & anisotropy & $\Delta \sigma$ & $\begin{array}{l}\delta \\
\text { CSGT } \\
\text { GIAOI } \\
\text { GAIM }\end{array}$ & $\eta$ & $\boldsymbol{\Omega}$ & $\hat{\mathbf{e}}$ \\
\hline \multirow[t]{3}{*}{$\mathrm{Co}(1)$} & -7827.8043 & 15.4186 & 15.4186 & 10.2791 & 0.734364 & 10.2791 & -0.39844 \\
\hline & -7827.8043 & 15.4186 & 15.4186 & 10.2791 & 0.734364 & 10.2791 & -0.39844 \\
\hline & -7860.7339 & 16.0271 & 16.0271 & 10.6848 & 0.851658 & 10.6848 & -0.22248 \\
\hline \multirow[t]{3}{*}{$C(2)$} & 35.7915 & 349.7468 & 349.7469 & 233.1646 & 0.001602 & 233.1646 & -1.4976 \\
\hline & 35.7915 & 349.7468 & 349.7469 & 233.1646 & 0.001602 & 233.1646 & -1.4976 \\
\hline & 19.0814 & 373.804 & 373.804 & 249.2026 & 0.000786 & 249.2026 & -1.49882 \\
\hline \multirow[t]{3}{*}{$C(3)$} & 35.8802 & 349.5784 & 349.5784 & 233.0522 & 0.000275 & 233.0522 & -1.49959 \\
\hline & 35.8802 & 349.5784 & 349.5784 & 233.0522 & 0.000275 & 233.0522 & -1.49959 \\
\hline & 19.1472 & 373.7295 & 373.7295 & 249.153 & 7.14E-05 & 249.153 & -1.49989 \\
\hline \multirow[t]{3}{*}{$C(4)$} & 35.8283 & 349.6323 & 349.6323 & 233.0881 & 0.000297 & 233.0881 & -1.49956 \\
\hline & 35.8283 & 349.6323 & 349.6323 & 233.0881 & 0.000297 & 233.0881 & -1.49956 \\
\hline & 19.0861 & 373.7933 & 373.7934 & 249.1956 & 5.26E-05 & 249.1956 & -1.49992 \\
\hline \multirow[t]{3}{*}{$C(5)$} & 35.808 & 349.6633 & 349.6633 & 233.1089 & 0.000288 & 233.1089 & -1.49957 \\
\hline & 35.808 & 349.6633 & 349.6633 & 233.1089 & 0.000288 & 233.1089 & -1.49957 \\
\hline & 19.064 & 373.8239 & 373.8239 & 249.216 & 8.83E-05 & 249.216 & -1.49987 \\
\hline \multirow[t]{3}{*}{$C(6)$} & 35.8821 & 349.5573 & 349.5574 & 233.0382 & 0.000264 & 233.0382 & -1.4996 \\
\hline & 35.8821 & 349.5573 & 349.5574 & 233.0382 & 0.000264 & 233.0382 & -1.4996 \\
\hline & 19.1366 & 373.7248 & 373.7248 & 249.1499 & 0.000134 & 249.1499 & -1.4998 \\
\hline \multirow[t]{3}{*}{$C(7)$} & 35.7907 & 349.7474 & 349.7474 & 233.165 & 0.001602 & 233.165 & -1.4976 \\
\hline & 35.7907 & 349.7474 & 349.7474 & 233.165 & 0.001602 & 233.165 & -1.4976 \\
\hline & 19.0811 & 373.804 & 373.804 & 249.2026 & 0.000786 & 249.2026 & -1.49882 \\
\hline \multirow[t]{3}{*}{$N(8)$} & -33.9122 & 527.9381 & 527.9381 & 351.9587 & 0.000605 & 351.9587 & -1.49909 \\
\hline & -33.9122 & 527.9381 & 527.9381 & 351.9587 & 0.000605 & 351.9587 & -1.49909 \\
\hline & -43.2697 & 546.1166 & 546.1166 & 364.0778 & 6.54E-05 & 364.0778 & -1.4999 \\
\hline \multirow[t]{3}{*}{$\mathrm{N}(9)$} & -34.1079 & 528.12 & 528.12 & 352.08 & 0.001431 & 352.08 & -1.49785 \\
\hline & -34.1079 & 528.12 & 528.12 & 352.08 & 0.001431 & 352.08 & -1.49785 \\
\hline & -43.363 & 546.2229 & 546.2229 & 364.1486 & 0.000444 & 364.1486 & -1.49933 \\
\hline \multirow[t]{3}{*}{$N(10)$} & -34.1006 & 528.0967 & 528.0967 & 352.0645 & 0.000689 & 352.0645 & -1.49897 \\
\hline & -34.1006 & 528.0967 & 528.0967 & 352.0645 & 0.000689 & 352.0645 & -1.49897 \\
\hline & -43.3832 & 546.2333 & 546.2334 & 364.1556 & 0.000105 & 364.1556 & -1.49984 \\
\hline \multirow[t]{3}{*}{$N(11)$} & -34.1084 & 528.1192 & 528.1192 & 352.0794 & 0.00143 & 352.0794 & -1.49786 \\
\hline & -34.1084 & 528.1192 & 528.1192 & 352.0794 & 0.00143 & 352.0794 & -1.49786 \\
\hline & -43.3629 & 546.2218 & 546.2219 & 364.1479 & 0.000443 & 364.1479 & -1.49934 \\
\hline \multirow[t]{3}{*}{$\mathrm{N}(12)$} & -34.063 & 528.0556 & 528.0557 & 352.0371 & 0.00073 & 352.0371 & -1.49891 \\
\hline & -34.063 & 528.0556 & 528.0557 & 352.0371 & 0.00073 & 352.0371 & -1.49891 \\
\hline & -43.3477 & 546.193 & 546.193 & 364.1286 & $3.05 \mathrm{E}-05$ & 364.1286 & -1.49995 \\
\hline \multirow[t]{3}{*}{$N(13)$} & -33.9229 & 527.9167 & 527.9167 & 351.9444 & 0.000552 & 351.9444 & -1.49917 \\
\hline & -33.9229 & 527.9167 & 527.9167 & 351.9444 & 0.000552 & 351.9444 & -1.49917 \\
\hline & -43.2617 & 546.0694 & 546.0694 & 364.0463 & 7.72E-05 & 364.0463 & -1.49988 \\
\hline
\end{tabular}




\section{CONCLUSION}

In this study, Density functional theory calculations with EPR basis sets have been employed to determination non-bonded interaction. In accordance with Table 3, definition the magnitude of " under strong field ligands, such as (CN); , equal to 0.25308 a.u. . In accordance with Table 1 , $\left[\mathrm{Co}(\mathrm{CN})_{6}\right]^{3-}$ complex with strong field ligands no exhibit the Jahn-Teller distortion. It has been displayed at Table 5 that the bonding coefficients of $s, p$ and $d$ orbitals were 0.3 and anti-bonding coefficients of $\mathrm{Co}-\mathrm{C}$ bonds were 0.8 and $\mathrm{Co}-\mathrm{N}$ bonds were 0.7 . In accordance with NICS values of Table 6, it's displayed that loops 1,3 and 5 have similar NICS values and equal to -10.1058 and loops 2,4 and 6 have similar NICS values and equal to -10.1189 . So, the more negative NICS values, the aromaticity and magnetism that loops most. According to different NMR methods of Table 9, characterize that CSGT and GIAO methods have similar quantity.

\section{ACKNOWLEDGMENTS}

The authors gratefully acknowledge the financial and other support of this research, provided by the Islamic Azad University, Eslamshahr Branch,Tehran,Iran.

\section{REFERENCES}

1. Wu, H.S.; Jiao, H.J. Chem. Phys. Lett. 2004, 386, 369-372

2. Wu, H.S.; Xu, X.H.; Strout, D.L.; Jiao, H.J .J Mol Model. 2005, 12, 1-8

3. Rogers, K.M.; Fowler, P.W.; Seifert, G. Chem. Phys. Lett. 2000, 332, 43-50

4. Zhu, H.Y.; Schmalz, T.G.; Klein, D.J. Int. J. Quantum Chem. 1997, 63, 393-401

5. Manolopoulos, D.E.; Fowler, P.W. Chem Phys Lett. 1991, 187, 1-7

6. Zope, R.R.; Dunlap, B.I. Chem. Phys. Lett. 2004, 386,403-407
7. Zhang.R.; Huyskensd T.Z.; Ceulemeans, A. Chem. Phys. 2005, 316, 35 - 44

8. Amiri. A.; Monajjemi. M.; Ketabi. S. Phys. Chem. Liq. 2007, 45, 425-433

9. Monajjemi, M.; Azad, M.T.; Haeri, H.H.; Zare, K.; Hamedani, Sh. J. Chem. Res. 2003, 2003, 454-456.

10. Reed, A. E.; Weinhold, F. J. Am. Chem. Soc.1985, 107, 1919-1921

11. Monajjemi, M.; Khaleghian, M. J. Cluster Sci. 2011, 22, 673-692 